\title{
Induced Hall-Like Current by Acoustic Phonons in Semiconductor Fluorinated Carbon Nanotube
}

\author{
Daniel Sakyi-Arthur ${ }^{1}$, Samuel Y. Mensah', Kofi W. Adu2,3*, Kwadwo A. Dompreh', \\ Raymond Edziah ${ }^{1}$, Natalia G. Mensah ${ }^{4}$, Cynthia Jebuni-Adanu ${ }^{5}$ \\ ${ }^{1}$ Department of Physics, College of Agriculture and Natural Sciences, U.C.C, Ghana \\ ${ }^{2}$ Department of Mathematics, College of Agriculture and Natural Sciences, U.C.C, Ghana \\ ${ }^{3}$ Department of Physics, Pennsylvania State University-Altoona College, Altoona, Pennsylvania, USA \\ ${ }^{4}$ Material Research Institute, Pennsylvania State University, University Park, Pennsylvania, USA \\ ${ }^{5}$ St. Francis College of Education, Hohoe, Volta Region, Ghana \\ Email: *cxa269@psu.edu
}

How to cite this paper: Sakyi-Arthur, D. Mensah, S.Y., Adu, K.W., Dompreh, K.A., Edziah, R., Mensah, N.G. and Jebuni-Adanu, C. (2020) Induced Hall-Like Current by Acoustic Phonons in Semiconductor Fluorinated Carbon Nanotube. World Journal of Condensed Matter Physics, 10, 71-87.

https://doi.org/10.4236/wjcmp.2020.102005

Received: November 5, 2019

Accepted: May 9, 2020

Published: May 12, 2020

Copyright $\odot 2020$ by author(s) and Scientific Research Publishing Inc. This work is licensed under the Creative Commons Attribution International License (CC BY-NC 4.0).

http://creativecommons.org/licenses/by/4.0/

\section{(c) (i) (8) Open Access}

\begin{abstract}
We show that Hall-like current can be induced by acoustic phonons in a nondegenerate, semiconductor fluorine-doped single-walled carbon nanotube (FSWCNT) using a tractable analytical approach in the hypersound regime $q \ell \gg 1$ ( $q$ is the modulus of the acoustic wavevector and $\ell$ is the electron mean free path). We observed a strong dependence of the Hall-like current on the magnetic field, $H$, the acoustic wave frequency, $\omega_{q}$, the temperature, $T$, the overlapping integral, $\Delta$, and the acoustic wavenumber, $q$. Qualitatively, the Hall-like current exists even if the relaxation time $\tau$ does not depend on the carrier energy but has a strong spatial dispersion, and gives different results compared to that obtained in bulk semiconductors. For $\omega_{q}=10^{12} \mathrm{~Hz}$ and $H=10^{6} \mathrm{~Wb} / \mathrm{m}^{2}$, the Hall-like current is $j_{y}^{A M E}=0.09 \mathrm{~A} \cdot \mathrm{cm}^{-2}$ in the absence of an electric field and $j_{y}^{A M E}=0.90 \mathrm{~A} \cdot \mathrm{cm}^{-2}$ in the presence of an electric field at $300 \mathrm{~K}$. Similarly, the surface electric field $E_{S A M E}$ due to the Hall-like current is $E_{S A M E}=0.21 \mathrm{~V} / \mathrm{m}$ in the absence of an external electric field. In the presence of an external electric field, $E_{S A M E}^{\max }=+0.74 \mathrm{~V} / \mathrm{m}$ and $E_{S A M E}^{\min }=-0.77 \mathrm{~V} / \mathrm{m}$ for $\boldsymbol{H}=15 \times 10^{6} \mathrm{~Wb} / \mathrm{m}^{2}$ at $300 \mathrm{~K} . q$ and $\Delta$ can be used to tune the Hall-like current and $E_{S A M E}$ of the FSWCNT. This offers the potential for room temperature application as an acoustic switch or transistor, as well as a material for ultrasound current source density imaging (UCSDI) and AE hydrophone device in biomedical engineering.
\end{abstract}




\section{Keywords}

Carbon Nanotube, Fluorinated, Hall-Like Current, Cyclotron

\section{Introduction}

Transport of electrons in semiconductor and multi-quantum well structures is governed by diffusive, drift and tunneling current flow [1]. In some cases, the materials exhibit ballistic transport provided the size of the system is smaller than the electron mean-free path, with the length ranging from nanometers to few microns as in some novel nanostructures [1]. Conceptually different from these mechanisms are transport phenomena based on energy and momentum exchange between externally propagating entities such as acoustic phonons and the electron medium. For most of the conduction electrons, their velocity component is much larger in magnitude than the speed of the acoustic wave, such that these electrons are "out of phase" with respect to the travelling electric field [2] [3]. Thus, the time average of the electric field over the trajectories of the electrons is zero, and that, these electrons are unaffected by the presence of the acoustic wave. However, there are few electrons that have velocity components that are parallel and comparable to the acoustic phonon wave velocity. These electrons are capable of being trapped by the moving electric field so that the time-average of their velocities in the direction of the field is exactly that of the field. Among these electrons, those having a maximum energy are found to give rise to a net electric current. In $n$-type semiconductors, these electrons are in the conduction band. This process of generating an electric current by the travelling acoustic wave is known as the acoustoelectric effect (AE) in the case of an open circuit or a constant electric field [1] [2] [3] [4].

In the presence of a weak magnetic field, $\Omega \tau \ll 1$ (where $\Omega$ is the cyclotron frequency and $\tau$ is the relaxation time), an acoustic wave propagating through a conductor also induces another type of effect called acoustomagnetoelectric effect (AME) [4]-[9]. AME current occurs when the sample is short circuited in the Hall direction and an AME field is induced if the sample is placed in a magnetic field stimulated by an acoustic wave propagating in the direction perpendicular to the magnetic field in an open circuit [5]-[9]. The AME current is similar to the Hall current generated in Hall effect where the acoustic wave plays the role of the electric field.

AME was first theorized by Grinberg et al. [10] in bipolar semiconductors and was experimentally observed a few years later in bismuth [11]. Epshtein et al. [12] relentlessly studied the effect in monopolar semiconductors. They observed that, the AME occurred as a result of the dependence of the electron relaxation time on the carrier energy, $\tau(\varepsilon)$, and that when $\tau$ is constant, the effect ceases to exist [12]. Subjecting a sample to a sound flux and an electric field along the $\mathrm{z}$-axis, and orienting the magnetic field parallel to the $\mathrm{x}$-axis and perpendicular 
to the sample, a non-zero current evolves in the Hall direction in an open circuit [5]-[9].

Modification of single-walled carbon nanotube (SWCNT) skeletal structure with fluorine is one of the emerging and efficient processes for chemical activation and functionalization of carbon nanotubes [13] [14]. Fluorination of multi-walled carbon nanotubes (FMWCNT) was performed decades ago, which was followed by SWCNTs in the latter years [15] [16]. Fluorination plays a significant role in the functionalization process as it provides a high surface concentration of functional groups, up to $\mathrm{C}_{2} \mathrm{~F}$ without destroying the tube's skeletal structure. The functionalization process is a fast exothermic reaction and the repulsive interactions of the fluorine atoms on the surface debundles the nanotube, thus, enhancing their electron dispersion [16].

Doping a SWCNT with fluorine atoms and thus, creating a double periodic band forms an FSWCNT, which modifies the metallic nature to semiconducting nature [17] [18]. Experimental measurements show that absorption of acoustic waves in FSWCNT is less than that of undoped SWCNT, and theoretical studies on the absorption of acoustic phonons in FSWCNT in the hypersound regime was done recently to confirm this [18] [19]. To the best of our knowledge, no studies of Hall-like current induced by acoustic phonons in FSWCNT with double periodic band in the hypersound regime have been reported. Therefore, the aim of this manuscript is to study the Hall-like current in FSWCNT as a function of the electric field, electron-phonon interaction, electron concentration, temperature, magnetic field and the acoustic wavenumber by employing a tractable analytical approach. For increasing the acoustic wavenumber, the electron concentration and the electron-phonon interaction, the acoustoelectric current is positive and increases in magnitude, but reverses direction depending on the external electric field. In the absence of the electric field, the Hall-like current rises to a resonance maximum and falls off in a manner similar to what has been observed in negative differential conductivity (NDC). Increasing temperature also decreases the net Hall-like current, however, that also rises and falls. We believe that the different behaviours due to the different acoustic wavelengths are characteristics of the different length scales over which the acoustic waves dictates the electronic properties of the semiconductor FSWCNT in the presence and absence of both external electric and magnetic fields.

\section{Theory}

Consider a fluorine-doped SWCNT, where the fluorine atoms form a one-dimensional chain along the axial direction, the energy band is deduced as [17] [18]:

$$
\varepsilon\left(\boldsymbol{p}_{z}\right)=\varepsilon_{o}+\Xi_{n} \Delta \cos ^{2 N-1}\left(a \boldsymbol{p}_{z}\right)
$$

where $a=\sqrt{3} b / 2 \hbar, \quad \Xi_{n}$ is a constant, $N$ is an integer and $\varepsilon_{o}$ is the minimum energy of the $\pi$-electrons within the first Brillouin zone. For $N=2$, the energy band for FSWCNT at the edge of the Brillouin zone is expressed as [17] [18]: 


$$
\varepsilon\left(\boldsymbol{p}_{z}\right)=\varepsilon_{o}+\Delta_{1} \cos \left(3 a p_{z}\right)+\Delta_{2} \cos \left(a p_{z}\right)
$$

where, $p_{o}$ is the quasi-momentum of an electron in the first Brillouin zone i.e. $-\pi / a \leq p_{o} \leq \pi / a$, with $\Delta_{1}=2 \Delta$, and $\Delta_{2}=6 \Delta$.

As we previously described [4], the phenomenon is similar to the thermoelectric effect where the net flow of heat along a temperature gradient is analogous to the net flow of travelling acoustic waves along the gradient. We calculate the combined AE-AME current density in FSWCNT using a tractable analytic model [4] [5]. Assume an array of FSWCNT forms a two-dimensional structure. The sound wave and the external electric field $\boldsymbol{E}(t)$ propagate along the FSWCNT axis (z-axis), and the magnetic field is directed parallel to the $\mathrm{x}$-axis. The Hall-like current is induced in the y-axis. The current density for such an orientation is given as:

$$
j=\frac{2 e}{(2 \pi \hbar)^{2}} \sum_{n, n^{\prime}} \int U_{n, n^{\prime}}^{a c} \Psi_{i}(\boldsymbol{p}, \boldsymbol{H}) \mathrm{d}^{2} \boldsymbol{p}_{z}
$$

where $\Psi_{i}\left(\boldsymbol{p}_{z}\right)$ is the solution to the Boltzmann kinetic equation in the absence of a magnetic field, and is expressed as:

$$
\boldsymbol{v} \frac{\partial \Psi_{i}}{\partial \boldsymbol{p}}+\boldsymbol{W}_{p}\{\Psi\}=v_{i}
$$

$\boldsymbol{p}_{z}$ is the electron momentum along the axial direction of the FSWCNT and $U_{n, n^{\prime}}^{a c}$ in Equation (3) is the electron-phonon interaction transition rate which is obtained by using the Fermi golden rule as in refs. [4] [5] [7] [9]:

$$
\begin{aligned}
U_{n, n^{\prime}}^{a c}= & \frac{2 \pi \Phi}{\omega_{q} v_{s}} \sum_{n, n^{\prime}}\left\{\left|G_{\boldsymbol{p}_{z}-\hbar q, \boldsymbol{p}_{z}}\right|^{2}\left[f\left(\varepsilon_{n}\left(\boldsymbol{p}_{z}-\hbar q\right)\right)-f\left(\varepsilon_{n}\left(\boldsymbol{p}_{z}\right)\right)\right] \delta\left(\varepsilon_{n}\left(\boldsymbol{p}_{z}-\hbar q\right)-\varepsilon_{n}\left(\boldsymbol{p}_{z}\right)+\hbar \omega_{q}\right)\right. \\
& \left.+\left|G_{\boldsymbol{p}_{z}+\hbar q, \boldsymbol{p}_{z}}\right|^{2}\left[f\left(\varepsilon_{n^{\prime}}\left(\boldsymbol{p}_{z}+\hbar q\right)\right)-f\left(\varepsilon_{n^{\prime}}\left(\boldsymbol{p}_{z}\right)\right)\right] \delta\left(\varepsilon_{n^{\prime}}\left(\boldsymbol{p}_{z}+\hbar q\right)-\varepsilon_{n^{\prime}}\left(\boldsymbol{p}_{z}\right)-\hbar \omega_{q}\right)\right\}
\end{aligned}
$$

Here $f\left(\boldsymbol{p}_{z}\right)=f\left(\varepsilon_{n, n^{\prime}}\left(\boldsymbol{p}_{z}\right)\right)$ is the unperturbed electron distribution function, $\Phi$ is the sound flux density, $\varepsilon_{n, n^{\prime}}\left(\boldsymbol{p}_{z}\right)$ is the electron energy band, $n$ and $n$ 'denote quantization of the electron energy band, and $G_{p_{z} \pm \hbar q, p_{z}}$ is the matrix element of the electron-phonon interaction. $\Psi_{i}$ is the root of the kinetic equation given as [4] [7]:

$$
\frac{e}{c}(\boldsymbol{v} \times \boldsymbol{H}) \frac{\partial \Psi_{i}}{\partial \boldsymbol{p}}+\boldsymbol{W}_{p}\{\Psi\}=v_{i},
$$

where $v_{i}$ is the electron velocity and $\boldsymbol{W}_{p}\{\ldots\}=(\partial f / \partial \varepsilon \ldots)^{-1} \boldsymbol{W}_{p}(\partial f / \partial \varepsilon)$. The operator $\boldsymbol{W}_{p}$ is a Hermitian operator and it is the collision operator describing the relaxation of the nonequilibrium distribution of the electron [5]. We assume $\tau$ is constant (the collision operator has the form $\boldsymbol{W}_{\boldsymbol{p}}=1 / \tau$ ) and a solution to Equation (6) as:

$$
\Psi_{i}=\Psi_{i}^{0}+\Psi_{i}^{1}+\Psi_{i}^{2}+\cdots
$$

Following the approach in ref. [5], we substitute Equation (7) into Equation (6) and solve by the method of iteration to obtain the zero approximation in the absence of the magnetic field ( $\boldsymbol{H}=0$ ) as: 


$$
\Psi_{i}^{0}=v_{i} \tau
$$

Similarly, in the first approximation, we obtain

$$
\Psi_{i}^{1}=-\frac{\tau^{2} e}{m c}(\boldsymbol{v} \times \boldsymbol{H})_{i}
$$

and $i=x, y, z$.

Substituting Equation (8) and Equation (9) into Equation (3) and using the principle of detailed balance, i.e. $\left|G_{p^{\prime}, p}\right|^{2}=\left|G_{p, p^{\prime}}\right|^{2}$, we obtain the net current density as:

$$
\begin{aligned}
j_{i}= & \frac{2 e}{(2 \pi \hbar)^{2}} \frac{2 \pi \Phi}{\omega_{q} v_{s}} \sum_{n, n^{\prime}} \int\left|G_{\boldsymbol{p}_{z}+\hbar q, \boldsymbol{p}_{z}}\right|^{2}\left[f\left(\varepsilon_{n^{\prime}}\left(\boldsymbol{p}_{z}\right)\right)-f\left(\varepsilon_{n^{\prime}}\left(\boldsymbol{p}_{z}+\hbar q\right)\right)\right] \\
& \times\left[\Psi_{i}\left(\boldsymbol{p}_{z}+\hbar q\right)-\Psi_{i}\left(\boldsymbol{p}_{z}\right)\right] \delta\left(\varepsilon_{n^{\prime}}\left(\boldsymbol{p}_{z}+\hbar q\right)-\varepsilon_{n^{\prime}}\left(\boldsymbol{p}_{z}\right)-\hbar \omega_{q}\right) \mathrm{d}^{2} \boldsymbol{p}_{z} \\
& -\frac{2 e}{(2 \pi \hbar)^{2}} \frac{2 \pi \Phi}{\omega_{q} v_{s}} \frac{e \tau^{2}}{m c} \sum_{n, n^{\prime}} \int\left|G_{\boldsymbol{p}_{z}+\hbar q, \boldsymbol{p}_{z}}\right|^{2}\left[f\left(\varepsilon_{n^{\prime}}\left(\boldsymbol{p}_{z}\right)\right)-f\left(\varepsilon_{n^{\prime}}\left(\boldsymbol{p}_{z}+\hbar q\right)\right)\right] \\
& \times\left[\Psi_{i}\left(\boldsymbol{p}_{z}+\hbar q\right)-\Psi_{i}\left(\boldsymbol{p}_{z}\right)\right] \delta\left(\varepsilon_{n^{\prime}}\left(\boldsymbol{p}_{z}+\hbar q\right)-\varepsilon_{n^{\prime}}\left(\boldsymbol{p}_{z}\right)-\hbar \omega_{q}\right) \mathrm{d}^{2} \boldsymbol{p}_{z}
\end{aligned}
$$

The matrix element of the electron-phonon interaction is given as:

$$
\left|G_{p^{\prime}, p}\right|=\frac{4 \pi e K}{\sqrt{2 \rho \omega_{q} \epsilon}}
$$

where $K$ is the piezoelectric modulus, $\epsilon$ is the lattice dielectric constant, and $\rho$ is the density of FSWCNT. $\Psi_{i}\left(\boldsymbol{p}_{z}\right)=l_{z}$, is the electron mean free path given as

$$
l_{z}=\tau v_{z}
$$

where

$$
v_{z}=\frac{\partial \varepsilon\left(\boldsymbol{p}_{z}\right)}{\partial \boldsymbol{p}_{z}}
$$

Substituting Equations (11)-(13) into Equation (10) yields:

$$
\begin{aligned}
j= & -\frac{2 K^{2} \pi \Phi e^{3} \tau \Theta\left(1-\alpha^{2}\right)}{\hbar^{3} \omega_{q}^{2} \epsilon \rho a \sqrt{1-\alpha^{2}}} \sum_{n, n^{\prime}} \int\left[f\left(\varepsilon_{n^{\prime}}\left(\boldsymbol{p}_{z}\right)\right)-f\left(\varepsilon_{n^{\prime}}\left(\boldsymbol{p}_{z}+\hbar q\right)\right)\right] \\
& \times\left[v_{z}\left(\boldsymbol{p}_{z}+\hbar q\right)-v_{z}\left(\boldsymbol{p}_{z}\right)\right] \delta\left(\varepsilon_{n^{\prime}}\left(\boldsymbol{p}_{z}+\hbar q\right)-\varepsilon_{n^{\prime}}\left(\boldsymbol{p}_{z}\right)-\hbar \omega_{q}\right) \mathrm{d} \boldsymbol{p}_{z} \\
& -\frac{2 K^{2} \pi \Phi e^{4} \tau^{2} \Theta\left(1-\alpha^{2}\right)}{\hbar^{3} \omega_{q}^{2} \epsilon \rho a \sqrt{1-\alpha^{2}} m c} \sum_{n, n^{\prime}} \int\left[f\left(\varepsilon_{n^{\prime}}\left(\boldsymbol{p}_{z}\right)\right)-f\left(\varepsilon_{n^{\prime}}\left(\boldsymbol{p}_{z}+\hbar q\right)\right)\right] \\
& \times\left[\left(\boldsymbol{v}\left(\boldsymbol{p}_{z}+\hbar q\right) \times \boldsymbol{H}\right)-\left(\boldsymbol{v}\left(\boldsymbol{p}_{z}\right) \times \boldsymbol{H}\right)\right] \delta\left(\varepsilon_{n^{\prime}}\left(\boldsymbol{p}_{z}+\hbar q\right)-\varepsilon_{n^{\prime}}\left(\boldsymbol{p}_{z}\right)-\hbar \omega_{q}\right) \mathrm{d}^{2} \boldsymbol{p}_{z}
\end{aligned}
$$

Under the orientation considered, the axial AE current $\left(j_{z}^{A E}\right)$ is given by the first term and its solution if found in [4]. The second term gives the Hall-like current $\left(j_{z}^{A M E}\right)$. The distribution function of the electrons in the presence of the applied electric field, $\boldsymbol{E}(t)$ is obtained by solving the Boltzmann transport equation in the $\tau$-approximation as

$$
\frac{\partial f(\boldsymbol{r}, \boldsymbol{p}, t)}{\partial t}+\boldsymbol{v}(\boldsymbol{p}) \cdot \nabla_{r} f(\boldsymbol{r}, \boldsymbol{p}, t)+e \boldsymbol{E} \nabla_{p} f(\boldsymbol{r}, \boldsymbol{p}, t)=-\frac{f(\boldsymbol{r}, \boldsymbol{p}, t)-f_{o}(\boldsymbol{p})}{\tau}
$$


which has a solution of the form:

$$
f\left(\boldsymbol{p}_{z}\right)=\int_{0}^{\infty} \frac{\mathrm{d} t^{\prime}}{\tau} \exp (-t / \tau) f_{o}\left(\boldsymbol{p}_{z}-e a \boldsymbol{E} t^{\prime}\right)
$$

where $f_{o}\left(\boldsymbol{p}_{z}\right)$ is the shifted Fermi-Dirac distribution given as:

$$
f_{o}\left(\boldsymbol{p}_{z}\right)=\frac{1}{\left[\exp \left(-\left(\varepsilon\left(\boldsymbol{p}_{z}\right)-\mu\right) / k T\right)+1\right]} .
$$

$\mu$ is the chemical potential which ensures the conservation of electrons, $k$ is the Boltzmann's constant, $T$ is the absolute temperature in energy units. Substituting Equation (16) and Equation (17) into Equation (14), we obtained the equations for $j_{z}^{A E}$ and $j_{y}^{A M E}$ which contain the Fermi-Dirac integral $\mathcal{F}_{1 / 2}$ of the order $1 / 2$ as:

$$
\begin{aligned}
j= & -\frac{2 K^{2} \pi \Phi e^{3} \tau \Theta\left(1-\alpha^{2}\right)}{\hbar^{3} \omega_{q}^{2} \epsilon \rho a \sqrt{1-\alpha^{2}}} \sum_{n, n^{\prime}} \int\left[\mathcal{F}_{1 / 2}\left(\varepsilon_{n^{\prime}}\left(\boldsymbol{p}_{z}\right)\right)-\mathcal{F}_{1 / 2}\left(\varepsilon_{n^{\prime}}\left(\boldsymbol{p}_{z}+\hbar \boldsymbol{q}\right)\right)\right] \\
& \times\left[v_{z}\left(\boldsymbol{p}_{z}+\hbar \boldsymbol{q}\right)-v_{z}\left(\boldsymbol{p}_{z}\right)\right] \delta\left(\varepsilon_{n^{\prime}}\left(\boldsymbol{p}_{z}+\hbar \boldsymbol{q}\right)-\varepsilon_{n^{\prime}}\left(\boldsymbol{p}_{z}\right)-\hbar \omega_{q}\right) \mathrm{d} \boldsymbol{p}_{z} \\
& -\frac{2 K^{2} \pi \Phi e^{4} \tau^{2} \Theta\left(1-\alpha^{2}\right)}{\hbar^{3} \omega_{q}^{2} \epsilon \rho a \sqrt{1-\alpha^{2}} m c} \sum_{n, n^{\prime}} \int\left[\mathcal{F}_{1 / 2}\left(\varepsilon_{n^{\prime}}\left(\boldsymbol{p}_{z}\right)\right)-\mathcal{F}_{1 / 2}\left(\varepsilon_{n^{\prime}}\left(\boldsymbol{p}_{z}+\hbar \boldsymbol{q}\right)\right)\right] \\
& \times\left[\left(\boldsymbol{v}\left(\boldsymbol{p}_{z}+\hbar \boldsymbol{q}\right) \times \boldsymbol{H}\right)-\left(\boldsymbol{v}\left(\boldsymbol{p}_{z}\right) \times \boldsymbol{H}\right)\right] \delta\left(\varepsilon_{n^{\prime}}\left(\boldsymbol{p}_{z}+\hbar \boldsymbol{q}\right)-\varepsilon_{n^{\prime}}\left(\boldsymbol{p}_{z}\right)-\hbar \omega_{q}\right) \mathrm{d}^{2} \boldsymbol{p}_{z}
\end{aligned}
$$

where

$$
\mathcal{F}_{1 / 2}\left(\eta_{f}\right)=\frac{1}{\Gamma(1 / 2)} \int_{0}^{\infty} \frac{\eta_{f}^{1 / 2} \mathrm{~d} \eta}{1+\exp \left(\eta-\eta_{f}\right)} .
$$

Here, $\left(\mu-\varepsilon_{c}\right) / k T \equiv \eta_{f}$ and $\Gamma(1 / 2)$ is the gamma function. For nondegenerate electron gas, where the Fermi level is several $k T$ below the energy of the conduction band edge $\varepsilon_{c},\left(k T \ll \varepsilon_{c}\right)$, the integral in Equation (19) approaches $\sqrt{\pi} / 2 \exp \left(\eta_{f}\right)$. The unperturbed distribution function from Equation (19) can be expressed as:

$$
f_{o}\left(\boldsymbol{p}_{z}\right)=A^{\dagger} \exp \left(-\left[\varepsilon\left(p_{z}\right)\right] / k T\right)
$$

where $A^{\dagger}$ is the normalization constant to be determined from the normalization condition $\int f(p) \mathrm{d} p=n_{o}$ as:

$$
A^{\dagger}=\frac{3 n_{o} a^{2}}{2 I_{o}\left(\Delta_{1}^{*}\right) I_{o}\left(\Delta_{2}^{*}\right)} \exp \left(\frac{\varepsilon_{o}-\mu}{k T}\right)
$$

$n_{o}$ is the electron concentration, and $I_{o}\left(\Delta_{x}^{*}\right)$ is the modified bessel function of zero order, where $x=1,2$. Assuming the electrons are confined to the lowest conduction band, that is $n=n^{\prime}=1$, then the quasi-velocity of the electrons in the system is also given as:

$$
v_{z}\left(\boldsymbol{p}_{z}\right)=-\left[3 a \Delta_{1} \sin \left(3 a \boldsymbol{p}_{z}\right)+a \Delta_{2} \sin \left(a \boldsymbol{p}_{z}\right)\right]
$$

In the absence of an external magnetic field, the axial $\mathrm{AE}$ current density in the first term of Equation (14) is deduced as: 


$$
\begin{aligned}
j_{z}^{A E}= & j_{z}^{A E}(0)\left\{1-4\left(\Delta_{z}^{*} \sin \left(\chi\left(1-\frac{v_{d}}{v_{s}}\right)\right) \cos B \sin \left(\frac{a}{2} \hbar q\right)\right.\right. \\
& \left.+\Delta_{s}^{*} \cos A \sin \left(3 \chi\left(1-\frac{v_{d}}{v_{s}}\right)\right) \sin \left(\frac{3}{2} a \hbar q\right)\right) \\
& \times \operatorname{coth}\left[\Delta_{s}^{*} \cos \left(3 \chi\left(1-\frac{v_{d}}{v_{s}}\right)\right) \cos A \cos \left(\frac{3}{2} a \hbar q\right)\right. \\
& \left.+\Delta_{z}^{*} \cos \left(\chi\left(1-\frac{v_{d}}{v_{s}}\right)\right) \cos B \cos \left(\frac{a}{2} \hbar q\right)\right] \\
& \times \operatorname{coth}\left[\Delta_{s}^{*} \cos \left(3 \chi\left(1-\frac{v_{d}}{v_{s}}\right)\right) \sin A \sin \left(\frac{3}{2} a \hbar q\right)\right. \\
& \left.\left.+\Delta_{z}^{*} \cos \left(\chi\left(1-\frac{v_{d}}{v_{s}}\right)\right) \sin B \sin \left(\frac{a}{2} \hbar q\right)\right]\right\}
\end{aligned}
$$

where

$$
\begin{aligned}
j_{z}^{A E}(0)= & j_{o}\left[\sinh \left\{\Delta_{s}^{*} \sin \left(\frac{3}{2} a \hbar q\right) \sin A+\Delta_{z}^{*} \sin \left(\frac{a}{2} \hbar q\right) \sin B\right\}\right. \\
& \left.\times \sinh \left\{\Delta_{s}^{*} \cos \left(\frac{3}{2} a \hbar q\right) \cos A+\Delta_{z}^{*} \cos \left(\frac{a}{2} \hbar q\right) \cos B\right\}\right]
\end{aligned}
$$

and

$$
j_{o}=\frac{4 A^{\dagger} \pi \Phi e^{3} K^{2} \tau \Theta\left(1-\alpha^{2}\right)}{\hbar^{3} \omega_{q}^{2} \epsilon^{2} \sigma a q \sqrt{1-\alpha^{2}}}, \chi=\hbar \omega_{q} a / v_{s}, \alpha=\omega_{q} / 12 \Delta a q
$$

Switching off the external electric field, Equation (23) then reduces to Equation (24). Similarly, the Hall-like current density in the second part of Equation (14) is obtained after some cumbersome calculations as:

$$
\begin{aligned}
& j_{y}^{A M E}=-\frac{2 A^{\dagger} K^{2} \pi \Phi e^{3} \tau^{2} \Theta\left(1-\alpha^{2}\right) \Omega}{\hbar^{3} \omega_{q}^{2} \epsilon \rho a \sqrt{1-\alpha^{2}}} \int_{0}^{\infty} \exp \left(-\frac{\mathrm{d} t^{\prime}}{\tau}\right) \\
& \times\left\{\sinh \left[\Delta_{1}^{*} \cos \left(3 e a E t^{\prime}\right) \sin A \sin \left(\frac{3}{2} a \hbar q\right)+\Delta_{2}^{*} \cos \left(e a E t^{\prime}\right) \sin B \sin \left(\frac{a}{2} \hbar q\right)\right]\right. \\
& \times \sinh \left[\Delta_{1}^{*} \cos \left(3 e a E t^{\prime}\right) \cos A \cos \left(\frac{3}{2} a \hbar q\right)+\Delta_{2}^{*} \cos \left(e a E t^{\prime}\right) \cos B \cos \left(\frac{a}{2} \hbar q\right)\right] \\
&-4\left(\Delta_{2}^{*} \sin \left(e a E t^{\prime}\right) \cos B \sin \left(\frac{a}{2} \hbar q\right)+\Delta_{1}^{*} \cos A \sin \left(3 e a E t^{\prime}\right) \sin \left(\frac{3}{2} a \hbar q\right)\right. \\
&\left.+\Delta_{1}^{*} \Delta_{2}^{*} \sin \left(e a E t^{\prime}\right) \sin \left(3 e a E t^{\prime}\right) \cos A \cos B \sin \left(\frac{a}{2} \hbar q\right) \sin \left(\frac{3}{2} a \hbar q\right)\right) \\
& \times \cosh \left[\Delta_{1}^{*} \cos \left(3 e a E t^{\prime}\right) \cos A \cos \left(\frac{3}{2} a \hbar q\right)+\Delta_{2}^{*} \cos \left(e a E t^{\prime}\right) \cos B \cos \left(\frac{a}{2} \hbar q\right)\right] \\
&\left.\times \cosh \left[\Delta_{1}^{*} \cos \left(3 e a E t^{\prime}\right) \sin A \sin \left(\frac{3}{2} a \hbar q\right)+\Delta_{2}^{*} \cos \left(e a E t^{\prime}\right) \sin B \sin \left(\frac{a}{2} \hbar q\right)\right]\right\}
\end{aligned}
$$


where $\boldsymbol{\Omega}=\mu \boldsymbol{H} / \hbar c, \Theta$ is the Heaviside step function, $\Delta_{1}^{*}=\Delta_{1} / k T$, $\Delta_{2}^{*}=\Delta_{2} / k T$. Simplifying further, we obtain

$$
\begin{aligned}
j_{y}^{A M E}= & j_{y}(0)\left\{1-4\left(\Delta_{2}^{*} \sin \left(\chi\left(1-\frac{v_{d}}{v_{s}}\right)\right) \cos B \sin \left(\frac{a}{2} \hbar q\right)\right.\right. \\
& \left.+\Delta_{1}^{*} \cos A \sin \left(3 \chi\left(1-\frac{v_{d}}{v_{s}}\right)\right) \sin \left(\frac{3}{2} a \hbar q\right)\right) \\
& \times \operatorname{coth}\left[\Delta_{1}^{*} \cos \left(3 \chi\left(1-\frac{v_{d}}{v_{s}}\right)\right) \cos A \cos \left(\frac{3}{2} a \hbar q\right)\right. \\
& \left.+\Delta_{2}^{*} \cos \left(\chi\left(1-\frac{v_{d}}{v_{s}}\right)\right) \cos B \cos \left(\frac{a}{2} \hbar q\right)\right] \\
& \times \operatorname{coth}\left[\Delta_{1}^{*} \cos \left(3 \chi\left(1-\frac{v_{d}}{v_{s}}\right)\right) \sin A \sin \left(\frac{3}{2} a \hbar q\right)\right. \\
& \left.\left.+\Delta_{2}^{*} \cos \left(\chi\left(1-\frac{v_{d}}{v_{s}}\right)\right) \sin B \sin \left(\frac{a}{2} \hbar q\right)\right]\right\}
\end{aligned}
$$

Switching off the external electric field from Equation (26) yields:

$$
\begin{aligned}
j_{y}(0)= & j_{o y}\left[\sinh \left\{\Delta_{1}^{*} \sin \left(\frac{3}{2} a \hbar q\right) \sin A+\Delta_{2}^{*} \sin \left(\frac{a}{2} \hbar q\right) \sin B\right\}\right. \\
& \left.\times \sinh \left\{\Delta_{1}^{*} \cos \left(\frac{3}{2} a \hbar q\right) \cos A+\Delta_{2}^{*} \cos \left(\frac{a}{2} \hbar q\right) \cos B\right\}\right]
\end{aligned}
$$

where

$$
j_{o y}=-\frac{4 e^{3} A^{\dagger} K^{2} \pi \Phi \tau^{2} \Theta\left(1-\alpha^{2}\right)}{\hbar^{4} \omega_{q}^{2} v_{s} \epsilon \rho a q \sqrt{1-\alpha^{2}}} \frac{\mu \boldsymbol{H}}{c}
$$

and

$$
A=\frac{3}{4} \sin ^{-1}\left(\frac{\omega_{q}}{12 \Delta a q}\right), B=\frac{1}{4} \sin ^{-1}\left(\frac{\omega_{q}}{12 \Delta a q}\right)
$$

The dependence of the Hall-like current density on $\omega_{q}, q$ and $T$ as expressed above is highly nonlinear.

\section{Results and Discussion}

The acoustic wave considered in this study is treated as packets of coherent phonons (monochromatic phonons) with wavelength $\lambda=2 \pi / q$, smaller than the mean free path of the FSWCNT electrons in the hypersound region $q l \gg 1$ ( $q$ is the modulus of the acoustic wavevector and $\ell$ is the electron mean free path).

Equations (23) and (26) and Equations (24) and (27) can be written in terms of the axial AE current as:

$$
j_{y}^{A M E}=j_{z}^{A E} \Omega \tau .
$$

The Hall-like current is proportional to the axial acoustoelectric current but 
depends on the dimensionless quantity $\Omega \tau$ which is a measure of the magnetic strength i.e. $j^{A M E} / j^{A E}=\Omega \tau$. It is therefore empirical that the existence of the Hall-like current in FSWCNT is due to the electron quantization and the non-parabolicity of the energy band, and not on the dependence of $\tau$ on $\varepsilon\left(\boldsymbol{p}_{z}\right)$ [3]. Equations (23), (26), (27), (29) and (30) are analyzed numerically using the following parameters: $\omega_{q}=10^{12} \mathrm{~s}^{-1}, v_{s}=2.5 \times 10^{3} \mathrm{~m} / \mathrm{s}, \Phi=10^{5} \mathrm{~Wb} / \mathrm{m}^{2}$, $\ell=10^{-5} \mathrm{~cm}$ and $q=10^{6} \mathrm{~cm}^{-1}$. From Equations (23) and (26), we plot the axial $\mathrm{AE}$ and the Hall-like current against $\left(1-v_{d} / v_{s}\right)$ for various $q$ values and also against $q$ for various temperatures $T$ values as shown in Figures 1 (a)-(d).

It can be inferred from Figure 1(a) and Figure 1(b) that, when the electric field is positive the current rises, reaches a maximum then falls off as in negative differential conductivity. On the other hand, when the electric field is negative the current decreases, reaches a minimum then increases until it saturates. This can be attributed to the Bragg's reflection at the band edges. Furthermore, the ratio of the height of the absorption peak to that of amplification peak for successive $q$ values differs by one. Increasing the $q$ tends to increase the axial current density $\left(j_{z}^{A E}\right)$ as shown in Figure 1(a), but the Hall-like current density $j_{y}^{A M E}$ decreases in the $y$-direction due to the increase in scattering along that direction (see Figure 1(b)). Though Bragg's reflection controls the transport process, increasing the temperature in the presence of the external electric field,

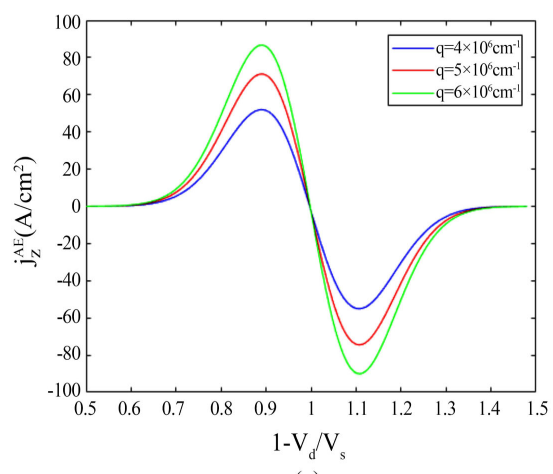

(a)

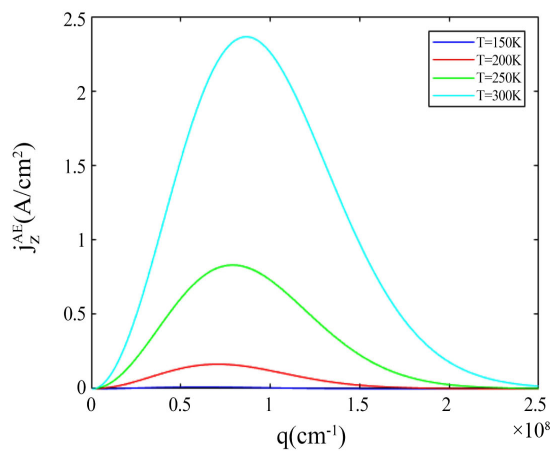

(c)

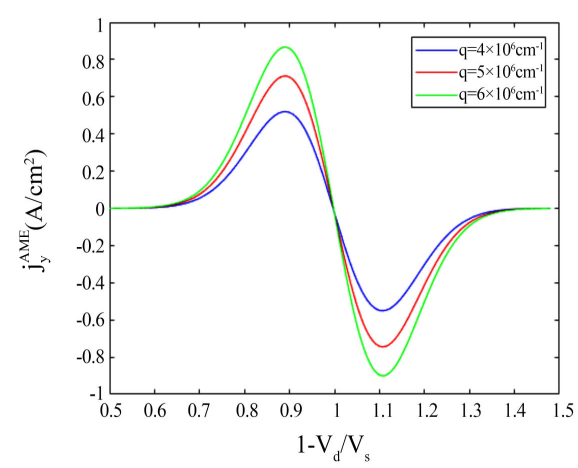

(b)

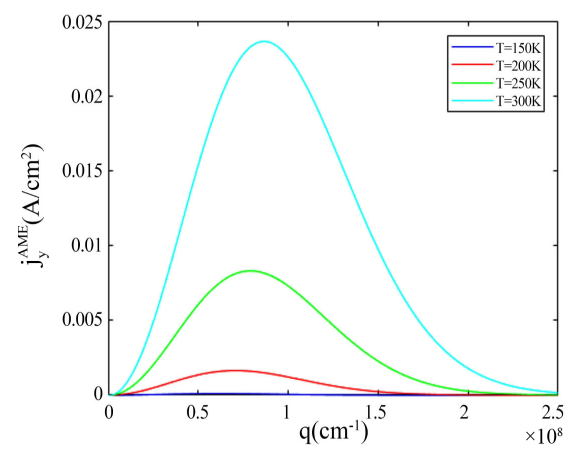

(d)

Figure 1. Dependence of (a) axial current $\left(j_{z}^{A E}\right)$, (b) Hall-like current $\left(j_{y}^{A M E}\right)$ on $1-v_{d} / v_{s}$ for different $q$ values at $T=300 \mathrm{~K}$ and $\Omega \tau=0.01$ (c) axial current density $j_{z}^{A E}$ (d) Hall-like current density $j_{y}^{A M E}$ on $q$ for different $T$ values at $\left(1-v_{d} / v_{s}\right)=0.5$. 
increases the conductivity as shown in Figure 1(c) and Figure 1(d), with the higher magnitude observed at room temperature $T=300 \mathrm{~K}$. This is because at near room temperatures, the band gap of the FSWCNT decreases, creating new conducting pathways where the majority of the electrons undergoes intraminiband transition. Consequently, a generation of high intraminiband current interacts strongly with the phonons to create a high $\mathrm{AE}$ and Hall-like current densities. In Figure $1(\mathrm{c})$, the AE current $j_{z}^{A E}$ is about two orders of magnitude greater than that of the Hall-like ( $j_{y}^{A M E}$ ) current (Figure $1(\mathrm{~d})$ ). This is due to the scattering of the induced Hall-like electrons.

Similarly, we display in Figure 2(a) and Figure 2(b), $j_{z}^{A E}$ and $j_{y}^{A M E}$, when the electric field is switched off. In the absence of the external electric field, there exists an inherent $d c$ field due to the phonons transferring their energy and momentum to the electrons so that the electrons move with a drift velocity, $v_{d}$, through the FSWCNT. The peak of $j_{z}^{A E}$ and $j_{y}^{A M E}$ in Figure 2(a) and Figure 2(b) decreases with increasing temperature, in contrast to what was observed in the presence of the external electric field. The electrons in this case have only a single path for conduction. Increasing the temperature, increases the kinetic energy of the electrons and the majority of these electrons undergo interminiband transition. Thus, only a handful of electrons undergo this intraminiband transition leading to the low conductivity as observed. Owing to the conservation laws of energy and momentum, it follows that only electrons with momentum $\boldsymbol{p}_{z}>\hbar q / 2$ interact with the acoustic phonons. Passing a high frequency sound flux through the sample, the absorption coefficient $\Gamma(q)$ as well as $j_{z}^{A E}$ and $j_{y}^{A M E}$ approach zero. Moreover, the nonlinear behaviour attributed to the external magnetic field, directs few electrons in the Hall direction which leads to weak interaction with the acoustic phonons.

In Figure 3, we show the dependence of $j_{z}^{A E}$ and $j_{y}^{A M E}$ on $T$ in the absence of the electric field. The $j_{z}^{A E}$ and $j_{y}^{A M E}$ rise linearly to a maximum and then begins to drop and shifts toward higher temperatures, and exhibit linear dependence on $T$ in the region of ohmic conductivity when $E=0$. This suggests that, increasing $q$ leads to a strong electron-phonon interaction where more phonons

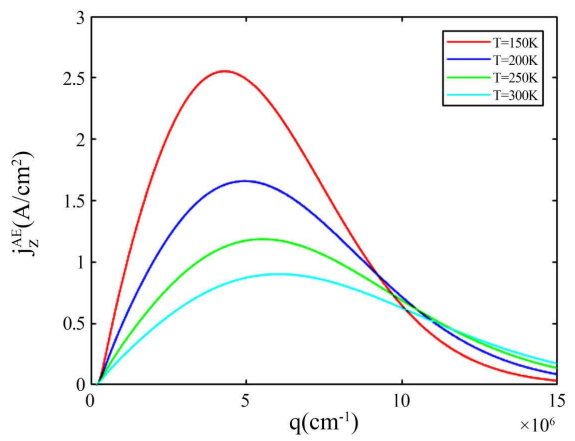

(a)

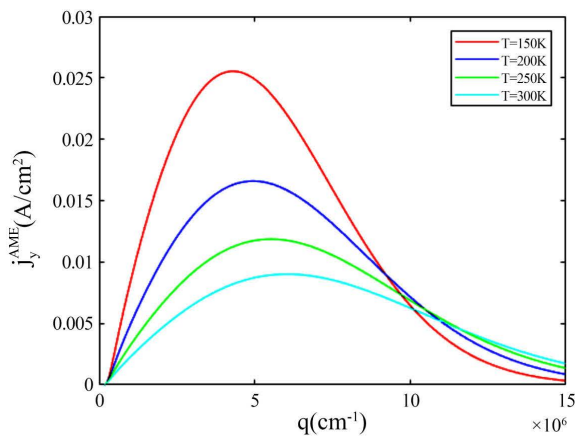

(b)

Figure 2. Dependence of (a) axial current $\left(j_{z}^{A E}\right)$ (b) Hall-like current $\left(j_{y}^{A M E}\right)$ on $q$ for different $T$ values at $\Omega \tau=0.01$. 


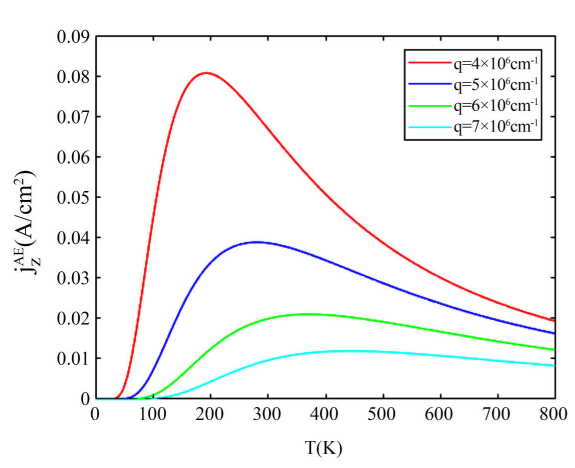

(a)

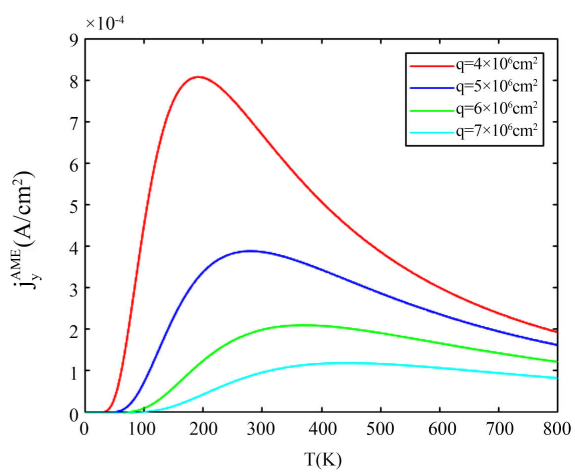

(b)

Figure 3. Dependence of (a) axial current $\left(j_{z}^{A E}\right)$ (b) Hall-like ( $j_{y}^{A M E}$ ) on $T$ for different $q$ values at $\Omega \tau=0.01$.

transfer their energy and momentum to the electrons to increase $j_{z}^{A E}$ and $j_{y}^{A M E}$. However, this is not the case at high temperatures because the energy of the electron-phonon interactions increases due to increase in their kinetic energies and collisions with other excitations. This leads to a handful of electrons undergoing intraminiband transition, leading to a decrease in both $j_{z}^{A E}$ and $j_{y}^{A M E}$. Therefore, there is a threshold temperature for which the $j_{z}^{A E}$ and $j_{y}^{A M E}$ turn on, suggesting that FSWCNT can be used as an acoustic switch or acoustic transistor. These occurs at $T=35 \mathrm{~K}$ for $q=4 \times 10^{6} \mathrm{~cm}^{-1}, T=56 \mathrm{~K}$ for $q=5 \times 10^{6} \mathrm{~cm}^{-1}, T=84 \mathrm{~K}$ for $q=6 \times 10^{6} \mathrm{~cm}^{-1}$ and $T=112 \mathrm{~K}$ for $q=7 \times 10^{6} \mathrm{~cm}^{-1}$. As shown in Figure 3(a) and Figure 3(b), $j_{z}^{A E} \gg j_{y}^{A M E}$ since the scattering is high along the Hall direction (y-axis).

In Figure 4, we display the dependence of $j_{z}^{A E}$ and $j_{y}^{A M E}$ on temperature for different values of electron-phonon interaction parameter, $\Delta$. As the temperature increases, the net $j_{z}^{A E}$ and $j_{y}^{A M E}$ remain zero, then rises to a maximum and decreases. The peak temperature shift towards higher temperatures with increasing $\Delta$. This suggests that higher currents are obtained at lower $\Delta$ values and low temperatures for both $j_{z}^{A E}$ and $j_{y}^{A M E}$. Therefore, $\Delta$ can be used to tune the current to high temperatures for room temperature applications as an acoustic switch or transistor. The shift towards higher temperatures in the current densities is attributed to the non-parabolicity of the dispersion relation which is very strong in FSWCNT. This accounts for the intersections of the curves for different values of $\Delta$ in $j_{z}^{A E}$ and $j_{y}^{A M E}$ (Figure 4(a) and Figure 4(b)). At these temperatures, different values of $\Delta$ have the same $q$ values and that, the same $j_{z}^{A E}$ and $j_{y}^{A M E}$. In Figure $4(\mathrm{~b})$, as the eletron-phonon interaction increases to $\Delta=0.10 \mathrm{eV}$, the peak of $j_{y}^{A M E}$ falls drastically due to increase in scattering along the Hall direction.

The relation between the attenuation coefficient, and the surface electric field due to the Hall-like current was proposed by Yamada [11] [20] as:

$$
\Gamma_{a b s} \Phi=\frac{n_{o} e E_{S A M E}}{\mu \boldsymbol{H} / \hbar c}
$$




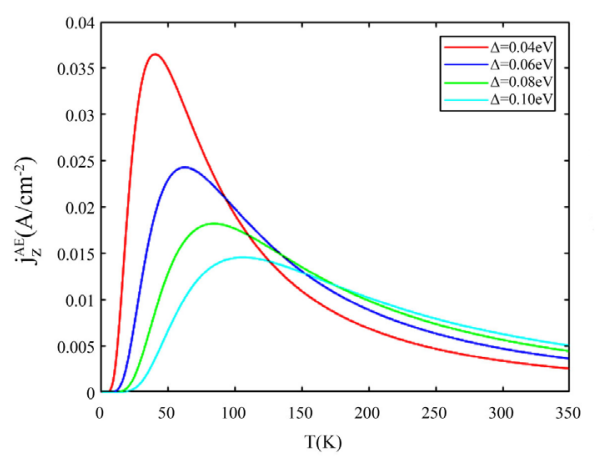

(a)

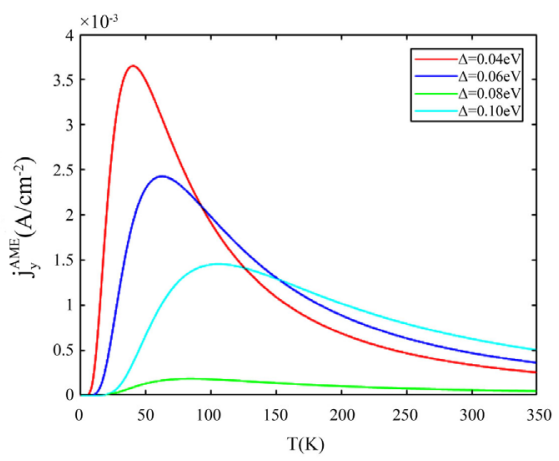

(b)

Figure 4. Dependence of (a) axial current $\left(j_{z}^{A E}\right)$ on $T(\mathrm{~b})$ Hall-like current $\left(j_{y}^{A M E}\right)$ on $T$ for different $\Delta$ values at $\Omega \tau=0.01$.

The absorption coefficient $\Gamma_{a b s}$ for FSWCNT is given in ref. [18] as:

$$
\begin{aligned}
\Gamma_{a b s}= & \frac{2 A^{\dagger} \pi \Phi^{2} K^{2} \Theta\left(1-\alpha^{2}\right)}{3 \hbar^{2} \omega_{q}^{2} \rho v_{s} \Delta \epsilon a q \sqrt{1-\alpha^{2}}} \\
& \times\left[\sinh \left\{\Delta_{1}^{*} \sin \left(\frac{3}{2} a \hbar q\right) \sin A+\Delta_{2}^{*} \sin \left(\frac{a}{2} \hbar q\right) \sin B\right\}\right. \\
& \left.\times \cosh \left\{\Delta_{1}^{*} \cos \left(\frac{3}{2} a \hbar q\right) \cos A+\Delta_{2}^{*} \cos \left(\frac{a}{2} \hbar q\right) \cos B\right\}\right]
\end{aligned}
$$

Thus, the surface electric field due to the Hall-like current $E_{S A M E}$ is expressed as:

$$
\begin{aligned}
E_{\text {SAME }}= & \frac{2 A^{\dagger} \pi \Phi^{2} K^{2} \Theta\left(1-\alpha^{2}\right)}{3 \hbar^{2} \omega_{q}^{2} \rho v_{s} \Delta \epsilon a q \sqrt{1-\alpha^{2}} n_{o} e}\left(\frac{\mu \boldsymbol{H}}{\hbar c}\right) \\
& \times\left[\sinh \left\{\Delta_{1}^{*} \sin \left(\frac{3}{2} a \hbar q\right) \sin A+\Delta_{2}^{*} \sin \left(\frac{a}{2} \hbar q\right) \sin B\right\}\right. \\
& \left.\times \cosh \left\{\Delta_{1}^{*} \cos \left(\frac{3}{2} a \hbar q\right) \cos A+\Delta_{2}^{*} \cos \left(\frac{a}{2} \hbar q\right) \cos B\right\}\right]
\end{aligned}
$$

The drift velocity of the electrons is given as:

$$
\begin{aligned}
v_{d}=\mu E_{\text {SAME }}= & \frac{2 A^{\dagger} \pi \Phi^{2} K^{2} \Theta\left(1-\alpha^{2}\right)}{3 \hbar^{2} \omega_{q}^{2} \rho v_{s} \Delta \epsilon a q \sqrt{1-\alpha^{2}} n_{o} e}\left(\frac{\mu^{2} \boldsymbol{H}}{\hbar c}\right) \\
& \times\left[\sinh \left\{\Delta_{1}^{*} \sin \left(\frac{3}{2} a \hbar q\right) \sin A+\Delta_{2}^{*} \sin \left(\frac{a}{2} \hbar q\right) \sin B\right\}\right. \\
& \left.\times \cosh \left\{\Delta_{1}^{*} \cos \left(\frac{3}{2} a \hbar q\right) \cos A+\Delta_{2}^{*} \cos \left(\frac{a}{2} \hbar q\right) \cos B\right\}\right]
\end{aligned}
$$

Thus, we display the dependence of the Hall-like field, $E_{\text {SAME }}$, on $q$ in the absence of an electric field in Figure 5 for different values of temperature (Figure 5(a)) and magnetic field (Figure 5(b)). Like Yamada [11], the calculated Hall-like field is highly nonlinearly dependent on $q$ (Figure 5) and linearly dependent on $H$ (Equation (31). As the temperature increases, the peak of the 


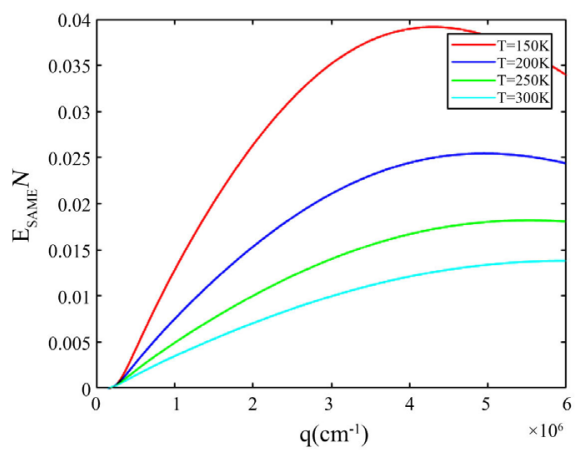

(a)

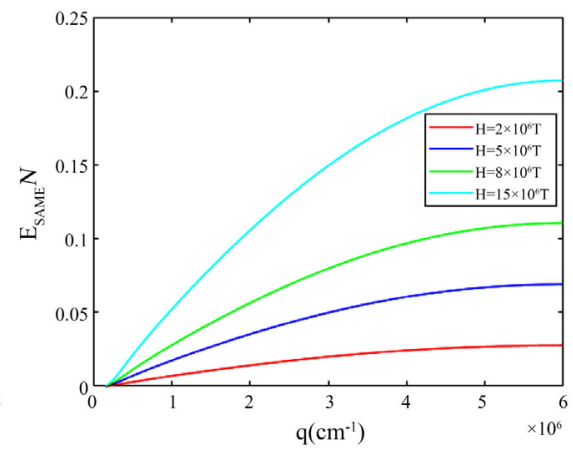

(b)

Figure 5. Dependence of Hall-like field $E_{S A M E}$ on wavenumber $(q)$ for varying (a) temperature (b) magnetic field $\boldsymbol{H}$.

Hall-like field decreases but shifts toward higher $q$ values (see Figure 5(a)). Note, the intensity of $E_{S A M E}$ decreases with increasing temperature. In Figure 5(b), the Hall-like field increases with increasing $q$ to a maximum and then falls off as $q$ increases. The intensity also increases with increasing magnetic field $\boldsymbol{H}$.

We display the dependence of the $E_{S A M E}$ on $1-v_{d} / v_{s}$ when the external electric field is switched on as calculated in Equation (31) (see Figure 6). It can be inferred that the orientation of the $H$-field produces a Hall-like field in the Hall direction which opposes the electron motion and thus, produces an absorption when $1 \ll v_{d} / v_{s}$. However, as $H$-field orientation reverses in the Hall direction and moves along with the electron motion with little opposition, thus, produces an amplification when $1 \gg v_{d} / v_{s}$. As the temperature increases, the peak of the Hall-like field decreases in both directions but shifts towards higher $1-v_{d} / v_{s}$ values (see Figure 6(a)). This is very interesting because, at $T=300 \mathrm{~K}$ increasing the magnitude of the $H$-field produces a high Hall-like field which will drive the electrons for a higher Hall-like current (see Figure 6(b)). In the presence of a magnetic field, the threshold electric field for amplification is increased due to the dc magnetoresistance. Furthermore, the magnetic field decreases the dielectric relaxation frequency and increases the diffusion frequency. The presence of a transverse magnetic field greatly reduces the electron drift velocity necessary to form the acoustoelectric domains [21] [22] [23] [24].

To put the results in perspective, we show in Figure 7(a) and Figure 7(b) a 3-D plot of $j_{z}^{A E}$ and $j_{y}^{A M E}$ against $q$ and (T). The $j_{z}^{A E}$ is about two orders of magnitude higher than the $j_{y}^{A M E}$. This is due to the weak field conditions $\Omega \tau \ll 1$.

\section{Conclusion}

We have shown that Hall-like current and field can be induced by acoustic phonons in a nondegerate, semiconductor FSWCNT using a tractable analytical approach in the hypersound regime $q \ell \gg 1$. A strong nonlinear dependence of Hall-like current $j_{y}^{A M E}$ and the field $E_{S A M E}$ on $H, q, T$ and $\Delta$ are observed. 


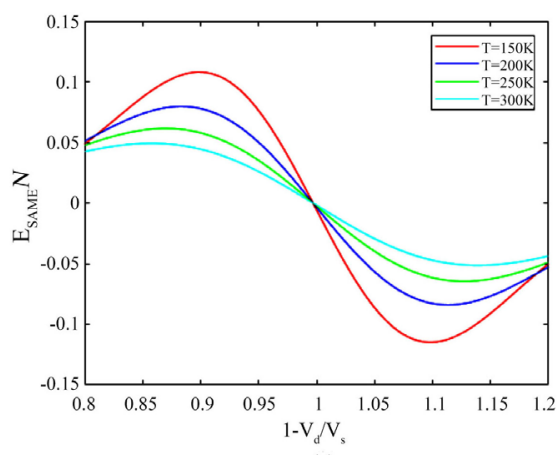

(a)

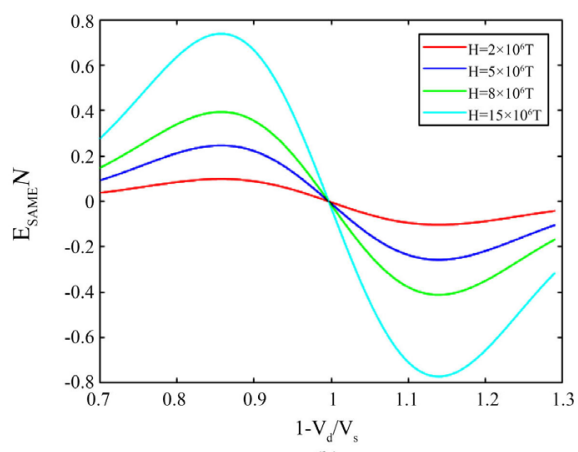

(b)

Figure 6. Dependence of Hall-like field $E_{S A M E}$ on electric field $1-v_{d} / v_{s}$ for varying (a) temperature $T(\mathrm{~b})$ magnetic field $H$.

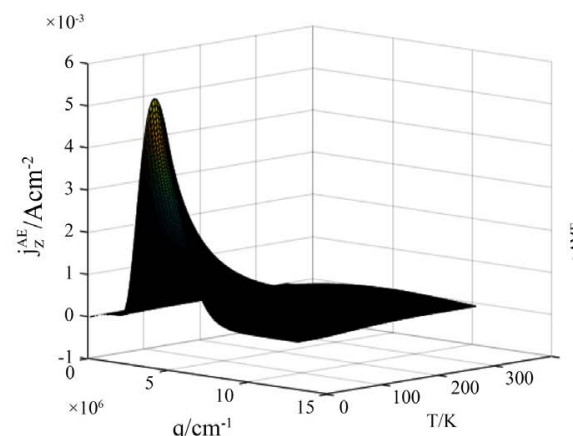

(a)

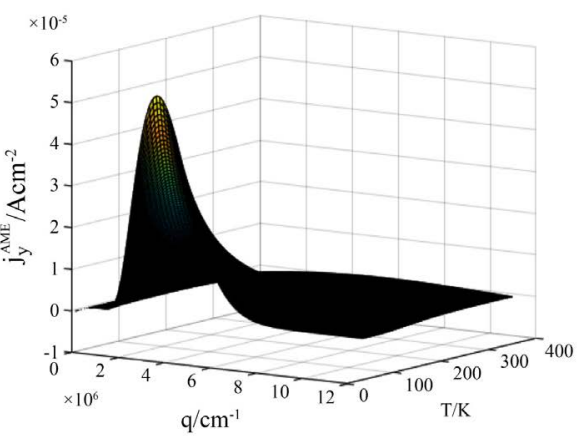

(b)

Figure 7. Dependence of (a) axial current $\left(j_{z}^{A E}\right)$ on $T(\mathrm{~b})$ Hall-like $\left(j_{y}^{A M E}\right)$ on $T$ and $q$.

This is due to the attenuation of acoustic phonons by electric field driven electrons experiencing intraminiband transition. Qualitatively, Hall-like current and field exist even if $\tau$ does not depend on the carrier energy but has a strong spatial dispersion. This result is different from that obtained in bulk semiconductors. In the case when $\tau$ is constant, the effect is only present in non-degenerate electron gas but absent in degenerate electron gas. These results suggest that FSWCNT could offer a huge potential for room temperature applications, however, novel techniques are needed in reducing its high electrical resistance. The acoustic wavenumber and the overlapping integral can be used to tune the Hall-like current and field of the FSWCNT for room temperature applications such as acoustic switch or transistor and also as a material for ultrasound current source density imaging (UCSDI) and AE hydrophone devices in biomedical engineering.

\section{Conflicts of Interest}

The authors declare no conflicts of interest regarding the publication of this paper.

\section{References}

[1] Lilly, M.P., Eisenstein, J.P., Pfeiffer, L.X. and West, K.W. (1998) Coulomb Drag in 
the Extreme Quantum Limit. Physical Review Letters, 80, 1714-1717. https://doi.org/10.1103/PhysRevLett.80.1714

[2] Gokhale, V.J., Shim, Y. and Rais-Zadeh, M. (2010) Observation of the Acoustoelectric Effect in Gallium Nitride Micromechanical Bulk Acoustic Filters. Frequency Control Symposium, Newport Beach, 1-4 June 2010, 524-529. https://doi.org/10.1109/FREQ.2010.5556273

[3] Epshtein, E.M. and Gulyaev, Y.V. (1967) AE Effect in Cds Pole Semiconductor. Soviet Physics, Solid State, 9, 288.

[4] Sakyi-Arthur, D., Mensah, S.Y., Adu, K.W., Dompreh, K.A., Edziah, R. and Mensah, N.G. (2020) Acoustoelectric Effect in Fluorinated Carbon Nanotube in the Absence of External Electric Field. World Journal of Condensed Matter Physics, 10, 1-11. https://doi.org/10.4236/wjcmp.2020.101001

[5] Mensah, S.Y., Allotey, F.K.A. and Adjepong, S.K. (1996) Acoustomagnetoelectric Effect in a Superlattice. Journal of Physics. Condensed Matter, 8, 1235. https://doi.org/10.1088/0953-8984/8/9/014

[6] Dompreh, K.A., Mensah, S.Y., Abukari, S.S., Edziah, R., Mensah, N.G. and Quaye, H.A. (2015) Acoustomagnetoelectric Effect in Graphene Nanoribbon in the Presence of External Electric and Magnetic Fields. Nanoscale Systems. Mathematical Modeling, Theory and Applications, 4, 50-55. https://doi.org/10.1515/nsmmt-2015-0005

[7] Mensah, S.Y., Allotey, F.K.A. and Adjepong, S.K. (1996) Acoutomagnetoelectric Effect in a Superlattice. Journal of Physics. Condensed Matter, 8, 1235-1239. https://doi.org/10.1088/0953-8984/8/9/014

[8] Margulis, A.D. and Vl Margulis, A. (1994) The Quantum Acoustomagnetoelectric Effect Due to Rayleigh Sound Waves. Journal of Physics. Condensed Matter, 6, 6139. https://doi.org/10.1088/0953-8984/6/31/013

[9] Mensah, N.G. (2006) Acoustomagnetoelectric Effect in Degenerate Semiconductor with Non-Parabolic Energy Dispersion Law.

[10] Grinberg, A.A. and Kramer, N.I. (1964) Acousto-Magnetic Effect in Piezoelectric Semiconductors. Doklady Akademii Nauk SSSR, 157, 79.

[11] Yamada, T. (1965) Acoustomagnetoelectric Effect in Bismuth. Journal of the Physical Society of Japan, 20, 1424-1437. https://doi.org/10.1143/JPSJ.20.1424

[12] Kogami, M. and Tanaka, S. (1971) Acoustomagnetoelectric and Acoustoelectric Effects in n-InSb at Low Temperatures. Journal of the Physical Society of Japan, 30, 775-784. https://doi.org/10.1143/JPSJ.30.775

[13] Khabashesku, V.N., Billups, W.E. and Margrave, J.L. (2002) Fluorination of Single-Wall Carbon Nanotubes and Subsequent Derivatization Reactions. Accounts of Chemical Research, 35, 1087-1095. https://doi.org/10.1021/ar020146y

[14] Bettinger, H.F. (2003) Experimental and Computational Investigations of the Properties of Fluorinated Single? Walled Carbon Nanotubes. ChemPhysChem, 4, 1283-1289. https://doi.org/10.1002/cphc.200300854

[15] Nakajima, T., Kasamatsu, S. and Matsuo, Y. (1996) Synthesis and Characterization of Fluorinated Carbon Nanotube. European Journal of Solid State and Inorganic Chemistry, 33, 831-840.

[16] Mickelson, E.T., Huffman, C.B., Rinzler, A.G., Smalley, R.E., Hauge, R.H. and Margrave, J.L. (1998) Fluorination of Single-Wall Carbon Nanotubes. Chemical Physics Letters, 296, 188-194. https://doi.org/10.1016/S0009-2614(98)01026-4

[17] Sadykov, N.R., Kocherga, E.Yu. and Dyachkov, P.N. (2013) Nonlinear Current in 
Modified Nanotubes with Exposure to Alternating and Constant Electric Fields. Russian Journal of Inorganic Chemistry, 58, 951-955. https://doi.org/10.1134/S0036023613080202

[18] Sakyi-Arthur, D., Mensah, S.Y., Mensah, N.G., Dompreh, K.A. and Edziah, R. (2018) Absorption of Acoustic Phonons in Fluorinated Carbon Nanotube with Non-Parabolic, Double Periodic Band. In: Phonons in Low Dimensional Structures, InTech, London, 129-142. https://doi.org/10.5772/intechopen.78231

[19] Jeon, T.-I., Son, J.-H., An, K.H., Lee, Y.H. and Lee, Y.S. (2005) Terahertz Absorption and Dispersion of Fluorine-Doped Single-Walled Carbon Nanotube. Journal of Applied Physics, 98, 34316-34316.

[20] Ohashi, Y.F., Kimura, K. and Sugihara, K. (1981) Acoustomagnetoelectric Effect in Graphite. Physica $B+C$, 105, 103-106. https://doi.org/10.1016/0378-4363(81)90224-2

[21] Abdelraheem, S.K., Blyth, D.P. and Balkan, N. (2001) Amplification of Ultrasonic Waves in Bulk GaN and GaAlN/GaN Heterostructures. Physica Status Solidi $(A)$, 185, 247-256. https://doi.org/10.1002/1521-396X(200106)185:2<247::AID-PSSA247>3.0.CO;2-H

[22] Hutson, A.R. and White, D.L. (1962) Elastic Wave Propagation in Piezoelectric Semiconductors. Journal of Applied Physics, 33, 40-47. https://doi.org/10.1063/1.1728525

[23] Abe, Y. and Mikoshiba, N. (1968) Ultrasonic Amplification in a Transverse Magnetic Field. Japanese Journal of Applied Physics, 7, 881. https://doi.org/10.1143/JJAP.7.881

[24] Kikuchi, M., Hayakawa, H. and Abe, Y. (1966) Acoustoelectric Current Oscillation in InSb and Its Dependence on the Transverse Magnetic Field. Japanese Journal of Applied Physics, 5, 1259. https://doi.org/10.1143/JJAP.5.1259 


\section{Nomenclature}

\begin{tabular}{|c|c|}
\hline Lattice type & Discrete lattice velocities \\
\hline$q$ & acoustic wavenumber \\
\hline$\ell$ & electron mean free path \\
\hline$j_{y}^{A E}$ & acoustoelectric effect current density \\
\hline$j_{y}^{A M E}$ & acoustomagnetoelectric effect current density/Hall-like current density \\
\hline $\boldsymbol{H}$ & magnetic field \\
\hline $\boldsymbol{E}_{\text {SAME }}$ & surface acoustomagnetoelectric field/Hall-like field \\
\hline$\omega_{q}$ & acoustic wave frequency \\
\hline $\boldsymbol{E}$ & constant electric field \\
\hline$\Omega$ & cyclotron frequency \\
\hline$\tau$ & relaxation time approximation \\
\hline$\varepsilon\left(p_{z}\right)$ & energy band relation \\
\hline$p_{z}$ & axial quasi-momentum \\
\hline$N, n$ & integer \\
\hline$\Delta$ & overlapping integral \\
\hline$p_{o}$ & quasi-momentum in the first brillouin zone \\
\hline$\hbar$ & reduced Planck constant \\
\hline$e$ & electronic charge \\
\hline$c$ & speed of light \\
\hline$v_{s}$ & speed of sound \\
\hline$v_{d}$ & drift velocity \\
\hline$\mu$ & electron mobility \\
\hline$k$ & Boltzmann constant \\
\hline$U_{n, n}^{a c}$ & electron-phonon interaction term \\
\hline$\Phi$ & acoustic phonon flux density \\
\hline$\hbar \omega_{q}$ & phonon energy \\
\hline$f\left(p_{z}\right)$ & electron distribution function \\
\hline $\mathcal{F}_{1 / 2}$ & Fermi-Dirac integral \\
\hline$A^{\dagger}$ & normalization term \\
\hline$I_{o}$ & Bessel function of order zero \\
\hline$G_{p, p}$ & matrix element of the electron-phonon interaction \\
\hline$\rho$ & density o FSWCNT \\
\hline$K$ & piezoelectric modulus \\
\hline$\epsilon$ & lattice dielectric constant \\
\hline$\Theta(x)$ & step function \\
\hline
\end{tabular}

